\title{
Electro-Optical Detection of Charged Particles *
}

\author{
Y.K. Semertzidis ${ }^{a}$, V. Castillo ${ }^{a}$, L. Kowalski ${ }^{b}$, D.E. Kraus ${ }^{c}$, \\ R. Larsen ${ }^{a}$, D.M. Lazarus ${ }^{\mathrm{a}, 1}$, B. Magurno ${ }^{\mathrm{a}, 2}$, D. Nikas ${ }^{\mathrm{a}}$, \\ C. Ozben ${ }^{\mathrm{a}}$, T. Srinivasan-Rao ${ }^{\mathrm{a}}$, T. Tsang ${ }^{\mathrm{a}}$ \\ a Brookhaven National Laboratory, Upton, NY 11973, U.S.A. \\ ${ }^{\mathrm{b}}$ Montclair State University, Upper Montclair, NJ 07043 U.S.A. \\ ${ }^{\mathrm{c}}$ University of Pittsburgh, Pittsburgh PA, 15260 U.S.A.
}

\begin{abstract}
We have made the first observation of a charged particle beam by means of its electro-optical effect on the polarization of laser light in a $\mathrm{LiNbO}_{3}$ crystal. The modulation of the laser light during the passage of a pulsed electron beam was observed using a fast photodiode and a digital oscilloscope. The fastest rise time measured, $120 \mathrm{ps,}$ was obtained in the single shot mode and was limited by the bandwidth of the oscilloscope and the associated electronics. This technology holds promise for detectors of greatly improved spatial and temporal resolution for single relativistic charged particles as well as particle beams.
\end{abstract}

Key words: Electro-optical devices, Charged particle detectors, Charged particle tracking, Picosecond techniques

\section{Introduction}

An effort has been initiated to develop an ultra-fast charged particle detector based on the birefringence, i.e. the anisotropy of the index of refraction,

ऋ Work supported in part by the U.S. Department of Energy under Contract No. DE-AC02-98CH10886

1 Corresponding author. Tel.: +1-631-344-3959;fax: +1-631-344-5954

E-mail address: lazarus@sun2.bnl.gov

2 Deceased.

Preprint submitted to Elsevier Preprint 27 October 2018 
produced in an optical medium by the electric field of relativistic charged particles [1]. The anisotropy of the index of refraction implies different velocities of light propagation for orthogonal components of polarization, resulting in a phase difference between them which will produce an elliptical polarization in light that had initially been linearly polarized. The ellipticity is defined as the ratio of the semi-minor to the semi-major axis of the ellipse traced by the electric field vector projected on a plane perpendicular to the direction of propagation. The electro-optical effect in amorphous optical media is known as the Kerr effect [2] and is quadratic in the electric field $E, \phi=2 \pi K E^{2} d$, where $\phi$ is the phase difference induced between orthogonal components of the polarized light. $K$ is the Kerr coefficient and $d$ is the length of the electric field region experienced by the material.

In uniaxial crystals, the induced ellipticity is linear in the E-field, and is known as the Pockels effect [3]. The difference in indices of refraction along orthogonal axes, $\Delta n=P E$ where $\mathrm{P}$ is the Pockels constant and $\mathrm{E}$ is the electric field. For $\mathrm{LiNbO}_{3}$, the Pockels constant $P=31 \mathrm{pm} / \mathrm{V}$. The induced phase delay can be written as $\phi=2 \pi \Delta n d / \lambda=\pi\left(V / V_{\pi}\right)$ with $V$ the applied voltage and $V_{\pi}$ the voltage required for producing a phase shift between orthogonal components of polarization equal to $\pi$ radians. The maximum ellipticity of $\pi / \sqrt{2}$ is induced when the polarization is at $45^{\circ}$ with respect to the applied electric field direction.

Devices based on the Pockels effect have long been employed in the detection of fast transient electrical signals as well as for modulation of optical signals [4].

\section{Apparatus}

The $45 \mathrm{MeV}$ kinetic energy electron beam at the Brookhaven National Laboratory Accelerator Test Facility, containing up to $1 \mathrm{nC}$ in a diameter of approximately $1 \mathrm{~mm}$ with 10 ps duration and a repetition rate of $1.5 \mathrm{~Hz}$, was scanned over a range of distances of several millimeters from the crystal.

The passage of beam bunches was detected through the effect of their electric field on the polarization of the light in a commercially available $\mathrm{LiNbO}_{3}$ electro-optical modulator crystal. [5]. The optics setup is depicted schematically in Fig. 1 The $\mathrm{LiNbO}_{3}$ crystal was coupled to two $40 \mathrm{~m}$ long polarization maintaining fibers of 4 microns core diameter. The first of the fibers propagated polarized light of wavelength $\lambda=1.32 \mu \mathrm{m}$ from a $20 \mathrm{~mW} \mathrm{CW} \mathrm{Amoco}$ laser operating at approximately $10 \mathrm{~mW}$ and the second fiber returned the transmitted light to a quarter wave plate which transformed the induced ellipticity to a rotation of the initially linear polarization which had previously been nearly extinguished by the analyzer (crossed polarizer) in the absence of 


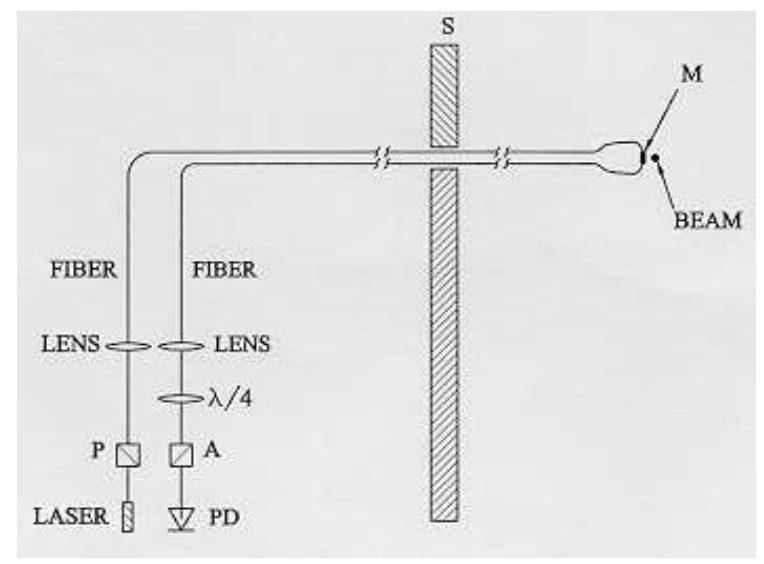

Fig. 1. The experimental setup for detecting a charged particle beam. The $\mathrm{LiNbO}_{3}$ crystal $(\mathrm{M})$ was located in vacuum several $\mathrm{mm}$ from the beam position which could be varied over several $\mathrm{cm}$. The beam direction was perpendicular to the plane of the page. The positions of the polarization maintaining fibers, polarizer $(\mathrm{P})$, lenses, $1 / 4$ wave plate $(\lambda / 4)$, analyzer $(\mathrm{A})$, shield wall $(\mathrm{S})$ and photodiode detector $(\mathrm{PD})$ are schematically indicated.

the electron beam. The resulting modulation of the transmitted laser light was detected by a photodiode and pre-amplifier of $45 \mathrm{GHz}$ bandwidth [6] which was followed by a $10 \mathrm{GHz}$ amplifier with $32 \mathrm{~dB}$ gain. The output signals were digitized in a $7 \mathrm{GHz}$ oscilloscope [7] and stored in memory.

The crystal modulator package had been modified to allow for the passage of the electron beam without hitting the housing. The transmitted light intensity, I, exiting the analyzer, A, is given by

$$
I=I_{0}\left[\sigma^{2}+(\alpha+\epsilon(t))^{2}\right] \approx I_{0}\left[\sigma^{2}+\alpha^{2}+2 \alpha \epsilon(t)\right]
$$

with $\epsilon$ the induced ellipticity, and $\alpha$ the misalignment angle. $\sigma^{2}$ is the minimum possible ratio of $\left(I / I_{0}\right)$ when $\alpha$ and $\epsilon$ are equal to zero and is defined as the extinction. A finite $\alpha$ can be used to linearize and amplify the effect on $\epsilon$. It is apparent from Eq. 1, the time dependent part of the light signal can be made positive or negative depending on the sign of $\alpha$ relative to $\epsilon$.

A bunched charged particle beam creates an electric field, $E$, at a distance $r$ that is well approximated by Coulomb's Law multiplied by $\gamma$, the relativistic Lorentz factor, and $N_{e}$ the number of electron charges in the beam bunch for a minimum distance $r_{0}$ much greater than the dimensions of the beam bunch.

$$
E=\gamma N_{e} \frac{q}{4 \pi \epsilon_{0} r_{0}^{2}}
$$

The electric field is present for $\Delta t=r_{0} / \gamma c$ with $r_{0}$ the effective range of the 
electric field, $E$, and $c$ the beam velocity. Laser light travels a distance

$$
\Delta l=(c / n) \Delta t=r_{0} / n \gamma
$$

in the crystal during the time that the electric field is present. This determines the resolving time for this technique. Since the beam was present in $10 \mathrm{ps}$ bunches, the photon detection system had insufficient high frequency bandwidth to avoid signal attenuation but the high intensity, $\approx 10^{10}$ electrons/ bunch, provided sufficient signal amplitude for detection.

The electric field sensitivity of the $\mathrm{LiNO}_{3}$ crystal was determined from the modulator specification provided by the manufacturer for the dependence of the phase difference on an applied high frequency voltage. For a phase difference $\pi$, the $\mathrm{LiNbO}_{3}$ crystal had $V_{\pi}=5.7 \mathrm{~V}$ with an electrode separation of $15 \mu \mathrm{m}$ and a length of $l=1.5 \mathrm{~cm}$. The integral

$$
\left(\int E d l\right)_{\pi}=\int 5.7 \mathrm{~V} /\left(15 \times 10^{-6} \mathrm{~m}\right) d l=5700 \mathrm{~V}
$$

produces the maximum ellipticity of $\pi / \sqrt{2}$.

The integral of the electric field of the particle bunch over the crystal dimensions for a beam bunch located at the mid-plane orthogonal to the length of the crystal at a distance $r_{0}$ is

$$
\int E d l \approx \frac{N_{e} q}{4 \pi \kappa \epsilon_{0} n r_{0}}
$$

where $\kappa$ is the dielectric constant and $\mathrm{n}$ is the index of refraction of the $\mathrm{LiNbO}_{3}$. For $r_{0}=5 \mathrm{~mm}, n=2.2$ and $\kappa=32$ for the electric field aligned with the $\mathrm{r}_{33}$ axis, $\int E d l=4.1 \times 10^{-9} N_{e} \mathrm{~V}$ which with Equation 4 yields an ellipticity

$$
\epsilon=\frac{\int E d l}{\left(\int E d l\right)_{\pi}} \times \pi / \sqrt{2}=7.2 \times 10^{-13} N_{e}
$$

The signal to noise ratio for a measurement of this type that is photon statistics limited is given by

$$
S N R=\epsilon \sqrt{\frac{P T q_{p}}{2 h \nu}}
$$

where $P$ is the laser power, $T$ the time resolution or inverse of the detection system bandwidth, $q_{p}$ the quantum efficiency of the photodiode, and $h \nu$ the laser photon energy. Excellent time resolution results in enhanced sensitivity, 


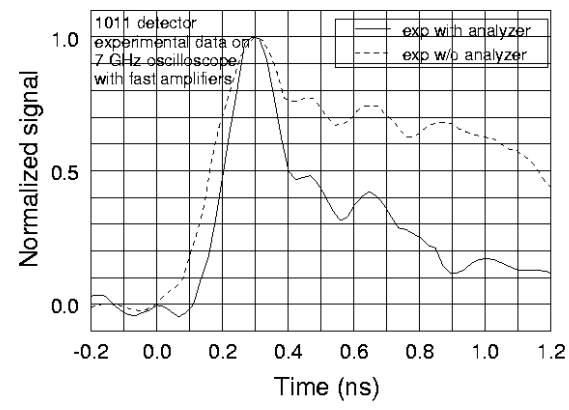

Fig. 2. The polarization dependent signal (solid line). The electron beam was $\approx 0.5 \mathrm{~cm}$ from the crystal. The polarization independent signal is indicated by the dashed line.

which can be essential for the detection of extremely short pulses, but it will also increase white noise acceptance due to the increased bandwidth.

\section{$3 \quad$ Experimental Results}

Measurements were made in the single shot mode using the $7 \mathrm{GHz}$ oscilloscope. The extinction was approximately $2 \times 10^{-3}$. With $P=10 \mathrm{~mW}, T=100 \mathrm{ps}$, $q_{p}=0.8$ and $h \nu=0.9 \mathrm{eV}$, Eqs.6 and 7 determine the required number of electrons in the beam for $S N R=1, N_{e} \approx 8 \times 10^{8}$ for detection of a single beam bunch. However, the $7 \mathrm{GHz}$ bandwidth limits the sensitivity to the inherently much faster signal by as much as factor of five implying $\approx 4 \times 10^{9}$ singly charged particles are required for detection of a single ATF beam bunch. The ATF beam of $N_{e}=6.3 \times 10^{9}$ per bunch was sufficient to generate a detectable modulation.

The polarization dependent signal is displayed in Fig. 2 (solid curve). Changing the sign of $\alpha$ (see Eq. 1) caused the sign of the signal to change whereas the amplitude was unaffected. The maximum modulation of the light intensity was $\approx 9 \%$ of its DC level.

A negative signal due to the attenuation of light was observed when the $\mathrm{LiNO}_{3}$ crystal intercepted the beam. It was found to be polarization independent and has a significantly longer decay time constant than the polarization dependent signal. A similar effect was found in poled quartz fibers and polarization main- 
taining fibers.

\section{Discussion}

Although it presently appears possible to construct a beam profile detector with excellent time resolution based on parallel rows of $\mathrm{LiNbO}_{3}$ crystals , a more formidable challenge would be to achieve single particle detection with this technology. With a high power pulsed laser of $10^{9} \mathrm{~W}$, i.e. $10 \mathrm{~mJ}$ for 10 ps and a transient digitizer of greater bandwidth, e.g. $60 \mathrm{GHz}$, a detector constructed of parallel rows of electro-optical crystals with a separation of $100 \mu \mathrm{m}$ and therefore an effective average distance, $r_{0} \approx 25 \mu \mathrm{m}$ between a single charged particle trajectory and the nearest fiber can be assumed. Equations 5 and 6 would then give an ellipticity $\epsilon=1.4 \times 10^{-10}$ and Equation 7 gives $S N R \approx 2.3 \times 10^{-2}$ for single particle detection with $\mathrm{LiNiO}_{3}$.

In addition, use of a high power pulsed laser would preclude the detection of randomly occurring events because of the inability to trigger the laser. However, this would be ameliorated somewhat at colliding beams machines where the beams are tightly bunched and interact periodically to maximize the luminosity.

The small size and high cost of the $\mathrm{LiNbO}_{3}$ crystals has prompted a search for materials of larger electro-optical coefficients and lower dielectric constants. We have measured the Kerr coefficients of optical fibers and quartz rods to be $10^{-4}-10^{-3} \mathrm{pm} / \mathrm{V}^{2}$. Organic liquids such as nitrobenzene have relatively large Kerr coefficients and could be used in hollow glass fibers of micron dimensions. Although these could lead to inexpensive devices, the Kerr coefficients are less than $2.5 \mathrm{pm} / \mathrm{V}^{2}$ which is far too small for sensitivity to singly charged particles.

Electrically poled optical fibers have been shown to produce relatively large electro-optical effects [8]. At sensitivities as high as $0.1 \mathrm{~nm} / \mathrm{V}^{2}$ they may be adequate for charged particle beam diagnostics but it is unlikely that it will be sufficiently sensitive for single particle detection.

DAST (4-dimethylamino-N-methyl-4-stilbazolium tosylate) crystals [9] have been found to have large electro-optic coefficients, from $47 \pm 8 \mathrm{pm} / \mathrm{V}$ at $\lambda=$ $1535 \mathrm{~nm}$ to $92 \pm 9 \mathrm{pm} / \mathrm{V}$ at $\lambda=720 \mathrm{~nm}$ as well as a small dielectric constant, $\kappa=5.2$ compared to $30.8 \mathrm{pm} / \mathrm{V}$ and $\kappa=32$ for $\mathrm{LiNbO}_{3}$. This implies a factor of 20 greater sensitivity than $\mathrm{LiNbO}_{3}$. If DAST or yet to be developed electro-

optical materials can yield adequate sensitivity for single particle detection, a new generation of ultrafast fine grained charged particle detectors may be at hand. 


\section{Acknowledgements}

We wish to acknowledge the support and encouragement of Xiejie Wang, Ilan Ben-Zvi, Vitaly Yakimenko, John Miller, Howard Gordon, Mike Murtagh and Tom Kirk. The efforts of Victor Usack and the contributions of our late colleague Ben Magurno were essential to our progress.

\section{References}

[1] Y.K. Semertzidis, XXVII International Conference on High Energy Physics gls0918 (1994)(Unpublished).

[2] J. Kerr, Phi. Mag. 50 (1875) 337, 446

[3] F. Pockels, Abhandl. Ges. Göttingen Math.-Phizik. Kl. 39 (1894) 1. A. Yariv, Quantum Electronics, Wiley, New York, 3rd ed. 1989.

[4] W. Willis, Proc. ECFA/INFN Workshop. CERN 85/07 (1985) 166 J.A. Valdmannis, G. Mourou and C.W. Gabel, IEEE J. Quantum Electronics QE19 (1983) 664. C. Bamber et al, Nucl. Instrum. Methods Phys. Res. Sect. A 327 (1993)227. T. Tsang and V. Radeka, Rev. Sci. Instrum. 66(7) (1995) 3844

[5] Uniphase Telecommunications Products, 1289 Blue Hills Ave., Bloomfield, CT 06002.

[6] Type 1011 pre-amplifier from New Focus, Inc., 2630 Walsh Ave., Santa Clara, CA 95051. The conversion gain of the detector is $10 \mathrm{~V} / \mathrm{W}$, with a rise time of 9 ps.

[7] The digitizing oscilloscope used was a Tektronix SDC 5000 of $7 \mathrm{GHz}$ bandwidth.

[8] X.C. Long and S.R.J. Brueck, IEEE Photonic Technol. Lett. 9 (1997) 767. David J. Welker et al., Opt. Lett. 23(23) (1998) 1826

[9] F. Pan et al., Appl. Phys. Lett. 69(1) (1996) 13 